\title{
Prevalence and Predictors of Anaemia Among Adolescent Girls in Rural Hadero Tunto District, Southern Ethiopia: Community Based Study
}

\author{
Tsegaye Alemu ${ }^{1, *}$, Samson Gebremedhin ${ }^{2, *}$ \\ ${ }^{1}$ Maternal and Child Health, John Snow Inc. Research \& Training Institute/Last Ten Killo Meters, South Nation National Region, Hawassa, \\ Ethiopia \\ ${ }^{2}$ Department of Public and Environmental Health, College of Health Science, Hawassa University, Hawassa, Ethiopia
}

Email address:

tsegayea49@gmail.com (T. Alemu)

${ }^{*}$ Corresponding author

\section{To cite this article:}

Tsegaye Alemu, Samson Gebremedhin. Prevalence and Predictors of Anaemia Among Adolescent Girls in Rural Hadero Tunto District, Southern Ethiopia: Community Based Study. International Journal of Nutrition and Food Sciences. Vol. 9, No. 1, 2020, pp. 16-24. doi: $10.11648 /$ j.ijnfs.20200901.14

Received: August 5, 2019; Accepted: March 2, 2020; Published: March 23, 2020

\begin{abstract}
Introduction: During adolescent anaemia has negative consequence on cognitive, work performance and economic productive. However, there is limited evidence on the prevalence and factors associated with anaemia among adolescent girls. Objectives: To assess the prevalence and associated factors of anaemia among rural adolescent girls in Hadero Tunto zuria district, Southern Ethiopia. Methods: A community based comparative cross-sectional study was conducted among adolescent girls 10-19 years. A total of 406 subjects were randomly selected. A structured questionnaire was used to collect the data. Blood haemoglobin level was determined from capillary blood using the Haemocue method. Data were entered into computer and analyzed using SPSS versions 20.0. Bivariate and multivariate logistic regression analyses were conducted to examine the factors associated with anaemia. Result: The mean $( \pm \mathrm{SD})$ of haemoglobin concentration adjusted for altitude was $13.7+1.2$ (SD) and ranged from 8 to $16.6 \mathrm{~g} / \mathrm{dl}$. Over all prevalence of anaemia was found to be $15.2 \%$ [ $95 \% \mathrm{CI}: 11.68 \%, 18.72 \%$ ]. Out of the total 406 adolescent girls $13.7 \%$ [95\% CI: $10.33 \%, 17.06 \%$ ] and 1.5\% [95\%CI: $0.3 \%, 2.7 \%$ ] had mild and moderate anaemia, respectively. Low dietary diversity, $(\mathrm{AOR}=10.0,95 \% \mathrm{CI}$ : 1.70, 60.0), long menstrual duration $(\mathrm{AOR}=5.10,95 \% \mathrm{CI}$ : $1.43,18.15)$, malaria attack history $(\mathrm{AOR}=3.50,95 \% \mathrm{CI}: 1.40,8.57)$, and less frequent consumption of legumes $(\mathrm{AOR}=3.20$, $95 \%$ CI: $1.17,8.74)$ were significantly associated with anaemia. Furthermore, the prevalence of stunting and thinness was $21.3 \%$ and $16.8 \%$, respectively. Conclusion: Anaemia is a public health problem in the study area; which require integrated governmental and NGO intervention to tackle the burden; enhance dietary diversity, promote Insecticide Treated Bednets (ITNs) utilization and adolescent nutrition education are important strategies to reduce the burden of anaemia.
\end{abstract}

Keywords: Rural Community, Adolescent Anaemia, Hadero Tunto District

\section{Introduction}

World Health Organization (WHO) defines 'adolescents' are individuals in the age range of $10-19$ years $[1,2]$. Adolescents are crucial period for growth and development, most of the existed literature witness adolescent is among high risk groups for iron deficiency Anaemia due to enhanced increase in requirements for iron, this due to rapid pubertal growth with sharp rise in lean body mass, blood volume and red cell mass, which increases iron needs for myoglobin in muscles and haemoglobin in the blood [3]. According to WHO [4] Iron-deficiency anaemia is one of the top five leading cause of global adolcescent disability adjusted life year (DALYS) lost, by sex and age group. Anaemia and under nutrition is a major public health problem among adolescent gilrs [5, 6]. Globally, the common cause of anaemia is iron deficiency, consequential from extended negative iron balance, affected by inadequate dietary iron intake and estimated $50 \%$ of anaemia in women worldwide is due to iron deficiency [7, 21]. In developing 
country, Anaemia affects large number of population [8]. Anaemia is a global public health problem with serious consequences for human health and development [21]. The widespread prevalence of Anaemia, both in the developed and developing worlds its of great concern [9]. In Africa a study report from 34 countries revealed that the estimated prevalence of anemia among adolescent and young females ranges from $15 \%$ to over $50 \%$ [10].

Even though recently additional emphasis is being given to the nutritional status of adolescents, most of the existing studies are limited to macro nutritional status. as initiatives gives attention only to prevent anaemia commonly target infants, young children and pregnant and lactating women, and not adolescents, the needs of adolescents may remain unmet, and the consequences of anaemia in adolescents continued. And also most of the existed studies are school and health facility based, so this study conducted at community level aims to assess the prevalence and associated factor of anaemia among adolescent girls.

\section{Methods and Materials}

Context: This study was conducted in Hadero Tunto Zuria (HTZ) district which was located $32 \mathrm{~km}$ from Zonal town Durame, $192 \mathrm{~km}$ from regional capital city of SNNPR Hawassa and $292 \mathrm{~km}$ from the capital city of Ethiopia Addis Ababa. HTZ district is one of the 7 districts which are found in Kembata Tembaro Zone. The district has 14 rural kebeles and 02 urban kebeles. Based on projection from 2007 Population and Housing Census report, the total population in $2014 / 15$ is estimated to be 127,059 among these 62,767 are males and 64,292 are females. From this, $74 \%$ of population resides in rural area and the rest $26 \%$ reside in town. Concerning to the health service facilities, there are 04 health centers and also 20 health posts on behalf of government and there are also 02 medium, 11 Junior clinic, 02 drug vendor and 02 drug store facilities owned by private owners. On the side of government, the health facilities covered by 10 health officers, 98 all types of nurses, 10 midwives and 48 health extension workers to access the service to the respective communities. From those 04 health centers $01 \mathrm{HC}$ is giving youth friendly service for adolescent and health coverage of the district was $90 \%$ [11].

Sample size: sample size was determined using single and double population proportion formula. Sample size was calculated by assuming the prevalence of adolescents girls anemia is $7.1 \%$ that was taken from previous study [12] with $95 \%$ confidence level, $3.5 \%$ margin of error and design effect of 1.5 .

For the first objective:

Assumption: Based on the previous study, the prevalence of anaemia among adolescent is 7.1\% [12]

Where: $n=$ Sample size, $\alpha=$ Level of significance (set at $0.05), \mathrm{z}=$ the standard normal deviate with $95 \%$ CI (1.96), $\mathrm{p}=$ Expected prevalence, $\mathrm{d}=$ Degree of precision $(0.035)$

$$
\mathrm{n}=\frac{(Z 1-\alpha / 2)^{2} p(1-p)}{d^{2}}
$$

$\mathrm{n}=(207+(207 * 10 \%$ non -response rate $)) * 1.5$ considering a design effect of 1.5 and a non-response rate of $10 \%$, the total sample size was

$\mathrm{n}=342$ adolescent girls.

For the second objective:

The sample size calculation for second objective, double population proportion formula used with Epi info version 7 software, with the assumption of $95 \% \mathrm{CL}, 80 \%$ power, $1: 1$ ratio of control to case and $10 \%$ non -respondent rate.

The first sample size which was 342, for adequacy, representativeness and to meet the objective 406 adolescent girls was recruited. In order to, assess the prevalence and associated factors of anaemia among adolescent girls.

Sampling technique: First of all, all kebeles in the district was listed and stratified into 3 ecological zones: Highland, midland and lowland. The sample size was allocated to the strata proportional to the population size. For each stratum 2 kebeles was selected at random. So, the sample size was distributed to the selected kebeles proportional to their population size. A sampling frame of adolescent girls were generated by identifying the number of adolescent girls living in each kebele using family folder (community health information system) from health extension workers who had the information in their respective kebeles. Then simple random sampling method was used to select 10-19 year adolescent girls from the frame.

Data collection Technique: structured pretested questionarie was used to collected data. The socio-demographic aspected of the questionnaire was taken from EDHS 2011 [13] and household dietary diversity (DD) was measured by using Food and Nutrition Techinical Assistance (FANTA) III tool and the DD was asked by using 24 hour recall method [14]. For that the subject should be asked whether she had taken any food from 12 food categories on the last day [15]. The questionnaire was developed based on the conceptual framework of the study. The questionnaire was developed in English and later translated into Amharic language and back translated to English to check its consistency.

The data collection was conducted by 4 clinical nurses, 1 laboratory technician and 1 laboratory technologist from the existing health system that were easily familiarize with the tool while conducting the training and actual data collection. The close follow-up by the very experienced supervisors, who was health professional from district health office after taking training about the purpose and method of administering the questionnaire together with data collectors. One immediate supervisor for three kebeles was assigned to the selected kebeles. Supervisors had checked whether the questionnaires were appropriately filled by the trained interviewers during the house-to-house survey. Adolescent girls were first informed about the study and its aim, and those agreeing to participate had given a written consent.

Haematology: Blood haemoglobin concentration was measured using a HemoCue $\mathrm{Hb} 301$ [16], (Angelhom, Sweden). Capillary blood was taken, through microcuvettes, and inserted into the HemoCue and the result was recorded, done by experienced laboratory technician. The necessary 
safety measures were taken during blood collection.

Measurements of height and weight were taken according to the WHO's guideline. Important anthropometric indicators were height-for-age z-scores (HAZ) and BMI z-scores (BMIZ) used and interpreted using age and sex specific cut off points recommended by WHO/CDC [17]. WHO reference 2007 used for interpretation [18] and WHO antroplus software used for assessing anthropometric data [19].

Data quality: the questionnaire was pretest on 20 adolescent girls those had similar socio-demographic characteristics out of study population. After pre-test, discussions were carried and based on the experience gained through the pre-test amendment to questionnaire missing part like skipping pattern, age category and food frequency questions were made correction. Data quality was ensured during instrument development, collection, coding, entry and analysis. The questionnaire first prepared in English and then translated into Amharic language and back translated to English in order to check its consistancy. Then data collectors were trained for a day about the purpose of the study and how to administer the questionnaire. Mock interviews and practical sessions on blood testing were also addressed in the training. During data collection, the questionnaire was checked for its completeness on daily basis by the supervisors. Incorrectly filled or missed questionnaires were returned back to the respective data collectors for correction. The investigator was also rechecking the completed questionnaires to maintain the quality of the data. There was discussion with data collectors and supervisors in every morning before the next day data collection. Data quality was also ensured during data coding, cleaning, entry to computer and during analysis.

Data Analysis: Data were checked manually for its completeness and consistency, entered into Statistical package for the social sciences (SPSS) versions 20.0 software, and cleaned by the principal investigator. Data were described and summarized using frequency, percentage, tables, graphs and measures of central tendency and dispersion. Bivitiate and multivariable logistic regression were applied to assess the association of socio-demographic and other independent factors with anemia. The outputs of the regression analysis were given with crude odds ratio and adjusted odds ratio with their respective confidence intervals. Criteria for exporting variables to the multivariate model were p-value less than 0.25 . Variables with P-value less than 0.05 were used to declare statistically significant association.

Ethical consideration: Ethical clearance was obtained from IRB of Hawassa University College of Medicine and Health Science and formal letters was written from regional health bureau to district. A written and informed consent and assent had been taken from the parents and adolescent girls, respectively. Those who weren't willing to participate had been given the right to do so. Confidentiality had been ensured.

\section{Result}

Of 406 adolescent girls recruited for the study, 400
(98.5\%) of the study subject participated voluntarily and as described in the method section the respondents were selected from 6 kebels. Most of the respondents, 393 $(98.0 \%)$, were rural residents. About 130 (32.5\%), 135 $(33.8 \%)$, and $135(33.8 \%)$ were selected from high, middle and lowlands, respectively. The altitude of the Kebeles included in the study ranged from 1518 to 2450 meters above sea level.

The mean $(+\mathrm{SD})$ age of the adolescent girls was $15.1( \pm 2.8$ $\mathrm{SD})$ years and ranged from 10 to 19 . About 167 (41.8\%) were in the early adolescent age group of 10-14 years, while $233(58.3 \%)$ were in the late adolescent age group of 15-19 years. As the educational background of the adolescent girls is concerned, $372(93.0 \%)$ had attended formal education, of which were $327(81.8 \%)$, and $45(11.3 \%)$ had attained primary and secondary education respectively, while the rest $28(7.0 \%)$ were illiterates.

Majority, $384(96.0 \%)$ of the study subject were single (unmarried). Regarding father educational status, illiterate fathers took the highest proportion, $213(53.3 \%)$ and, while the rest $129(32.3 \%)$ and $58(14.5 \%)$ were attended primary and secondary or above school, respectively. The majority of $333(83.3 \%)$ of father's occupation were farming and followed by merchant $48(12 \%)$, the rest $11(2.8 \%)$ were civil servant. More than half, $264(66.0 \%)$ of the respondents mother's had not attained formal education, which were illiterate. The rest $112(28.0 \%)$, and $24(6.0 \%)$ had attained the formal education in primary and secondary or above, respectively. Higher proportion $310(77.5 \%)$ of mothers were not involved in income generating activity (IGA), while the remaining $90(22.5 \%)$ had income generating activity.

The median family size was 7.0 ranged from 3 to 13 . Majority of the respondent, 241 (60.3\%) had 4-7 family size, about $18(4.5 \%)$ of the respondent had 1-3 family size, while the rest $141(35.3 \%)$ of the study participant had 8 or above family size respectively. Higher proportion of respondents $386(96.5 \%)$ were Protestant religion and the rest $11(2.8 \%)$ of the study subjects were orthodox religion. Regarding to the ethnic group distribution, more than half, $245(61.3 \%)$ were kembata and $64(16.0 \%)$ were Donga, while the rest 55 $(13.8 \%)$ and $16(4.0 \%)$ were Hadiya and Amahara, respectively (Table 1$)$.

Table 1. Socio-demographic characteristic of adolescent girls in HTZ district, KTZ, SNNPR, Ethiopia, 2016.

\begin{tabular}{lll}
\hline Variables & Frequency & Percentage \\
\hline Age $($ year $)(\mathrm{n}=400)$ & & \\
$10-14$ & 167 & 41.8 \\
$15-19$ & 233 & 58.3 \\
Family size $(\mathrm{n}=400)$ & & \\
$1-3$ & 18 & 4.5 \\
$4-7$ & 241 & 60.3 \\
$>8$ & 141 & 35.3 \\
Adolescent educational $(\mathrm{n}=400)$ & & \\
Illiterate & 28 & 7.0 \\
Primary $(1-8$ grade) & 327 & 81.8 \\
Secondary above & 45 & 11.3 \\
Marital status $(\mathrm{n}=400)$ & & \\
Single & 384 & 96.0 \\
\hline
\end{tabular}




\begin{tabular}{lll}
\hline Variables & Frequency & Percentage \\
\hline Others & 16 & 4.0 \\
Religion (n=400) & & \\
Protestant & 386 & 96.5 \\
Others & 14 & 3.5 \\
Father education (n=400) & & \\
Illiterate & 213 & 53.3 \\
Primary (1-8 grade) & 129 & 32.3 \\
Secondary or above & 58 & 14.5 \\
Mother education $(\mathrm{n}=400)$ & & \\
Illiterate & 264 & 66.0 \\
Primary (1-8 grade) & 112 & 28.0 \\
Secondary or above & 24 & 6.0 \\
Father occupation (n=400) & & \\
Farmer & 333 & 83.3 \\
Civil servant & 11 & 2.8 \\
Merchant & 48 & 12.0 \\
Others & 8 & 2.0 \\
Mother IGA (n=400) & & \\
Yes & 90 & 22.5 \\
No & 310 & 77.5 \\
Household wealth index (n=397) & & \\
Poor & 159 & 40.1 \\
Medium & 80 & 20.2 \\
Richer & 158 & 39.8 \\
Residence (n=400) & & \\
Rural & 393 & 98.3 \\
Urban & 7 & 1.8 \\
\hline
\end{tabular}

Knowledge about anemia

As described in the methods section, knowledge towards anemia was assessed using composite index. Only 29 (7.3\%) adolescent girls had good knowledge regarding anemia and $79(19.9 \%)$ of the study participant had average knowledge, while the rest $289(72.8 \%)$ of study subject had poor knowledge regarding anaemia (Figure 1).

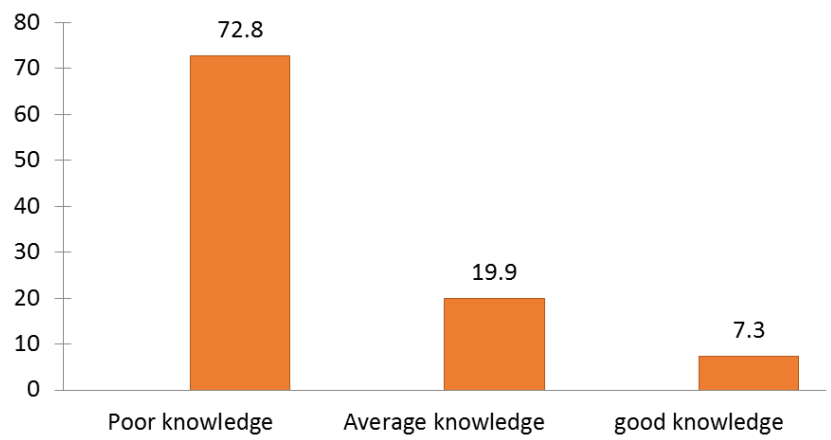

Figure 1. Frequency distribution of knowledge regarding anemia among adolescent girls in HTZ district, 2016.

Dietary intake of adolescents

The respondents were asked to describe their staple diet; most respondents mentioned maize based (180 (45\%)) and root based (133 (33.3\%)) foods; while Enset and teff based foods were mentioned by $65(16.3 \%)$ and $21(5.3 \%)$ of the respondents, respectively.

Based on FANTA score household DDS were calculated out of 12 food groups. The mean $( \pm \mathrm{SD})$ household DDS was $4.09( \pm 1.05)$ and ranged from 2 to 7 . Based on the cutoff points recommended by FAO, the majority $242(60.5 \%)$ had medium DD, 115 (28.8\%) of the study subjects had low and the remaining $43(10.8 \%)$ had high DD (Figure 2). Most of the adolescent girls, $347(86.8 \%)$ ate food made from root and followed by $295(73.8 \%)$ of cereals, $235(58.8 \%)$ of milk and milk product, $180(45.0 \%)$ of legumes, $114(28.5 \%)$ of oil or butter, $58(14.5 \%)$ of eggs, $34(8.5 \%)$ of meat and no one ate fresh or dried fish.

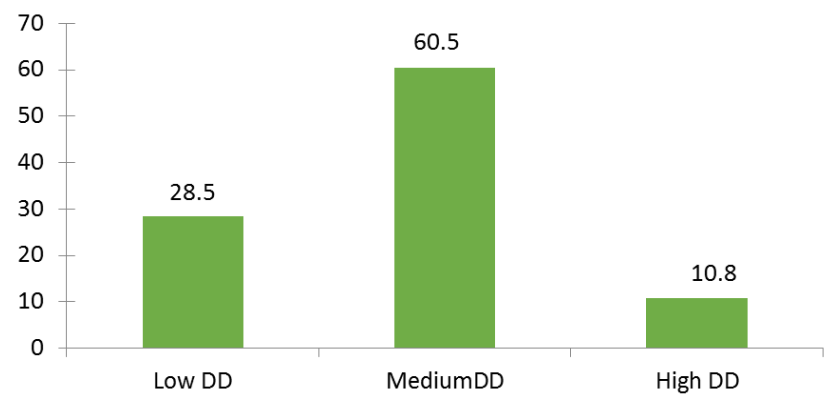

Figure 2. Frequency of dietary diversity score among adolescent girls in HTZ district, 2016.

The dietary intake pattern was also assessed using food frequency method. The mean $(+\mathrm{SD})$ frequency of meat consumption per week was $1.36(+0.54)$. Among the adolescent girls, $266(66.5 \%)$ of they had not eaten meat in the preceeding week, while the rest $123(30.8 \%)$ and $12(3.0 \%)$ had eaten 1-2times and $>3$ times per week, respectively. More than half, $214(53.5 \%)$ respondent ate greens leafy vegetables $>3$ times per week, about $141(35.3 \%)$ of the study participant had eaten green leafy vegetable 1-2 times per week, while the rest $45(11.3 \%)$ of the respondents had not eaten green leafy vegetables per week. The mean $(+\mathrm{SD})$ frequency of consumption of green leaf vegetable per week was 2.42 (+ 0.68). More than half, $225(56.3 \%)$ of the respondent had eaten legumes $>3$ times per week, $134(33.6 \%)$ the study subjects reported that as they ate legumes 1-2 times per week, while the rest $40(10.0 \%)$ adolescent girls had not eaten legumes per week (Table 2).

Table 2. Pattern of intake of selected food items for adolescent girls in rural HTZ district, KTZ, SNNPR, Ethiopia, 2016.

\begin{tabular}{llll}
\hline \multirow{2}{*}{ Food item } & \multicolumn{4}{l}{ Frequency of meal intake per week } \\
\cline { 2 - 4 } & No $(\%)$ & $\mathbf{1 - 2}$ times $\mathbf{( \% )}$ & > 3times $\mathbf{( \% )}$ \\
\hline Meat & 66.3 & 30.8 & 3.0 \\
Green vegetables & 11.3 & 35.3 & 53.5 \\
Legumes & 10.0 & 33.6 & 56.3 \\
\hline
\end{tabular}

Regarding adolescent dietary frequency, meal frequency was 3-4 times per day for the majority of adolescent girls 390 $(97.5 \%)$, but $10(2.5 \%)$ of the adolescent girls reported that they eat only 1-2 times a day. Furthermore, the study participant asked to describe the yesterday feeding frequency, only $26(6.5 \%)$ of the adolescent girls mentioned, as they ate 1-2 times per day, while the rest $374(93.5 \%)$ of study participant ate 3-4 times per day (Table 3).

Of 400 study subjects, $284(71.0 \%)$ of the respondents had coffee drinking habit after meals, of which $196(70.0 \%)$ consumed 2-4 cup, $52(18.6 \%)$ and $32(11.4 \%)$ of the respondents had consumed less than 2 cup and greater than 4 cup of coffee, respectively. 
Table 3. Frequency distribution of diet and health service related variables among Adolescent girl; HTZ district, KTZ, SNNPR, Ethiopia, 2016.

\begin{tabular}{|c|c|c|c|}
\hline Variable & & Frequency & Percentage \\
\hline \multicolumn{4}{|l|}{ Staple diet } \\
\hline & Maize based & 180 & 45.0 \\
\hline & Root based & 134 & 33.5 \\
\hline & Enset based & 65 & 16.3 \\
\hline & Teff based & 21 & 53 \\
\hline \multicolumn{4}{|c|}{ Dietary diversity } \\
\hline & High & 43 & 10.8 \\
\hline & Medium & 242 & 60.5 \\
\hline & Low & 115 & 28.5 \\
\hline \multicolumn{4}{|c|}{ Meal frequency/day in a typical day } \\
\hline & 1-2 times & 10 & 2.5 \\
\hline & 3-4 times & 390 & 97.5 \\
\hline \multicolumn{4}{|c|}{ Meal frequency/day in the preceeding day } \\
\hline & 1-2 times & 26 & 6.5 \\
\hline & 3-4 times & 374 & 93.5 \\
\hline \multicolumn{4}{|c|}{ Average coffee consumption per day (cups) } \\
\hline & $<2$ & 52 & 18.6 \\
\hline & $2-4$ & 196 & 70.0 \\
\hline & $>4$ & 32 & 11.4 \\
\hline
\end{tabular}

Prevalence of stunting and underweight among adolescents

Nutritional status of adolescent girls was evaluated by using BMI-for-age and height-for-age, the mean $( \pm \mathrm{SD}) \mathrm{z}-$ scores for the two indices were $-0.70 \pm 1.30$, and $-0.51 \pm 1.37$, respectively. Out of the study participant, 66 (16.5\%) had BMI-for-age less than -2SD, they were thinness, and 85 $(21.3 \%)$ of the respondent had height-for-age less than -2SD, indicating stunted (Figure 3).

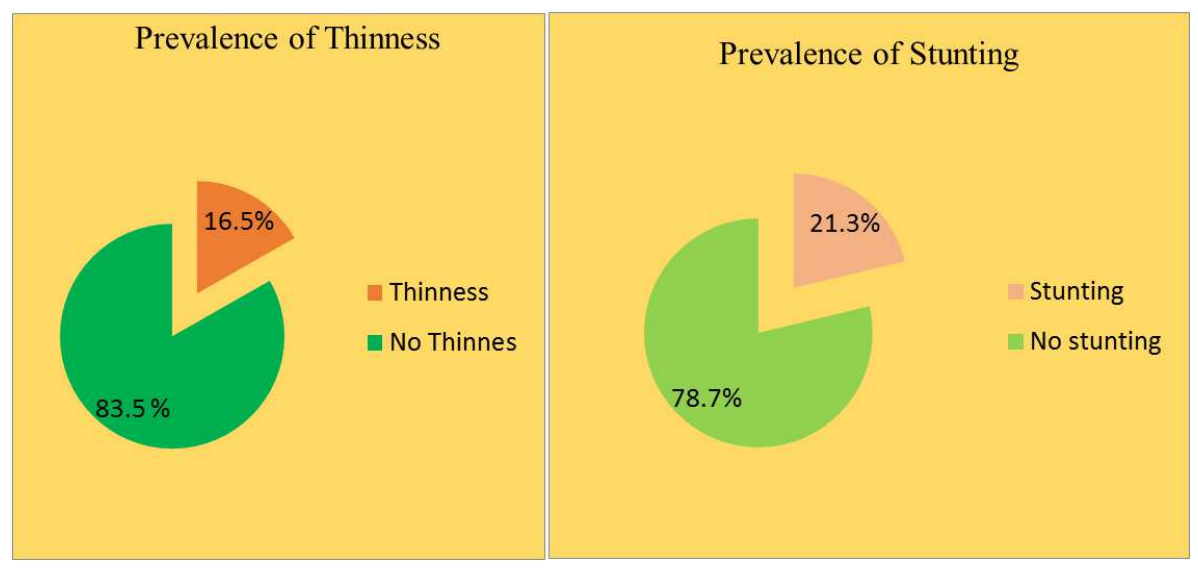

Figure 3. Distribution for prevalence of thinness and stunting among adolescent girls in HTZ district, 2016.

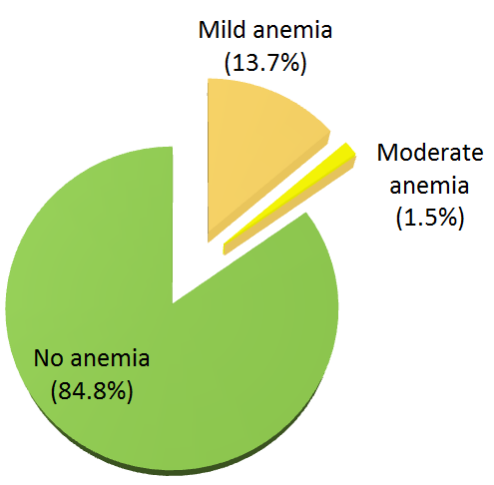

Figure 4. Distribution of prevalence of anaemia among adolescent girls in HTZ district, KTZ, SNNPR, 2016.

Prevalence of Anaemia

The mean $(+\mathrm{SD})$ of haemoglobin concentration adjusted for altitude [20] was $13.7+1.2$ and ranged from 8.0 to 16.6 $\mathrm{g} / \mathrm{dl}$. The overall prevalence of anemia was $15.2 \%$ [95\% CI: $11.7 \%, 18.7 \%$ ]. Out of the total 400 adolescent girls $13.7 \%$ [95\% CI: $10.3 \%, 17.1 \%]$ and $1.5 \%$ [95\% CI: $0.3 \%, 2.7 \%$ ] had mild and moderate anaemia, respectively. But there was no severe anaemia case in this study (Figure 4).

Factors association with anaemia

A total of 21 variables were considered for the bivaraite analysis. These include, age and educational status of the adolescent, father's and mother's educational status, father occupation, mothers involvement in IGA, family size, household wealth index, marital status of the adolescent, stunting, thinness, history of malaria infection, frequency of green vegetables, legumes and meat consumption, distance from nearby health facility, menstrual duration, heavy menstrual bleeding, physical workload, dietary diversity and knowledge on anemia. 
During the bivariate analysis 9 variables were found to have $p$ value less than 0.25 , these include, father occupation, history of malaria infection, frequency of meat consumption, frequency of legumes consumption, menstrual duration, physical workload, heavy menstrual bleeding, dietary diversity and knowledge regarding anemia, hence considered for the multivariate analysis.

However, after adjustment was made using multivariate model, low dietary diversity, $[\mathrm{AOR}=10.0,95 \% \mathrm{CI}: 1.70$, $60.0]$, long menstrual duration $[\mathrm{AOR}=5.10,95 \% \mathrm{CI}: 1.43$, $18.15)]$, malaria attack history $[\mathrm{AOR}=3.50,95 \% \mathrm{CI}: 1.40$, 8.57] and less frequently consumption of legumes [AOR=3.20, 95\%CI: 1.17, 8.74] showed significant association with anemia (Table 4).

The prevalence of anemia was higher among those who had low dietary diversity, as compare to their counterparts. Taking high DD group as a reference, the odds were 10.0 [95\% CI: 1.7, 60.0] times increased in the low DD category.
Similarly, those who consumed legumes less frequently (1-2 times /week) had 3.20 [AOR=3.20, 95\%CI: 1.17, 8.74] times increased odds of developing anemia, as compared to those who consume legumes three or more times per week. The proportion of anemia was higher among those who had history of malaria infection in the last 3 months, and the odds were 3.48 times higher in the group $[\mathrm{AOR}=3.48,95 \% \mathrm{CI}$ : $1.4,8.57]$.

In the bivariate analysis, adolescent girls who had heavy menstrual bleeding compared to light menses had increased odds of anemia. However, in multivariate logistic model the association failed to be significant. On the other hand, the relationship between menstrual duration and anemia risk was assessed. Less than 5 days considering as reference, the odds of being anemic were 5.10 times $[\mathrm{AOR}=5.10,95 \% \mathrm{CI}: 1.43$, 18.15] higher among adolescent girls whose menstrual duration stay 5-7 days than in those whose menstrual duration was less than 5 days (Table 4).

Table 4. Multivariate Logistic regression model analysis of predictors influencing anemia among adolescent girls rural HTZ district, KTZ, SNNPR, Ethiopia, 2016, $(n=400)$.

\begin{tabular}{|c|c|c|c|c|}
\hline \multirow{2}{*}{ Variables } & \multicolumn{2}{|c|}{ Anemia } & \multirow{2}{*}{ COR 95\% CI } & \multirow{2}{*}{ AOR 95\% CI } \\
\hline & Yes & No & & \\
\hline \multicolumn{5}{|l|}{ Father occupation } \\
\hline Others & 5 & 14 & $2.50[0.66,9.47]$ & $0.44[0.04,5.60]$ \\
\hline Farmers & 50 & 283 & $1.23[0.49,3.06]$ & $1.24[0.32,4.85]$ \\
\hline Merchant & 6 & 42 & 1 & 1 \\
\hline \multicolumn{5}{|l|}{ Knowledge score } \\
\hline Poor & 43 & 246 & $2.36[0.54,10.29]$ & $1.15[0.19,6.76]$ \\
\hline Average & 14 & 65 & $2.91[0.62,13.67]$ & $1.64[0.25,10.79]$ \\
\hline Good & 2 & 27 & 1 & 1 \\
\hline \multicolumn{5}{|c|}{ Frequency of legumes consumption/week } \\
\hline 0 & 9 & 31 & $2.04[0.88,4.74]$ & $2.87[0.77,10.70]$ \\
\hline 1-2 times & 23 & 111 & $1.46[0.80,2.65]$ & $3.20[1.17,8.74]^{*}$ \\
\hline$>3$ times & 28 & 197 & 1 & 1 \\
\hline \multicolumn{5}{|l|}{ Frequency of meat consumption/week } \\
\hline 0 & 40 & 225 & $0.36[0.10,1.24]$ & $0.40[0.03,4.83]$ \\
\hline 1-2 times & 17 & 106 & $0.32[0.09,1.18]$ & $0.62[0.50,7.58]$ \\
\hline$>3$ times & 4 & 8 & 1 & 1 \\
\hline \multicolumn{5}{|l|}{ Menstrual duration } \\
\hline 5-7 days & 18 & 12 & $12.35[5.35,28.49]^{*}$ & $5.10[1.43,18.15]^{*}$ \\
\hline$<5$ days & 26 & 214 & 1 & 1 \\
\hline \multicolumn{5}{|l|}{ Menstrual bleeding } \\
\hline Heavy & 22 & 23 & $6.76[3.08,14.80]^{*}$ & $2.60[0.83,8.23]$ \\
\hline Light & 23 & 203 & 1 & 1 \\
\hline \multicolumn{5}{|l|}{ Dietary diversity score } \\
\hline Low & 34 & 81 & $8.60[1.96,37.60]^{*}$ & $10.04[1.68,60.09]^{*}$ \\
\hline Medium & 24 & 217 & $2.27[0.52,9.97]$ & $2.85[0.50,17.9]$ \\
\hline High & 2 & 41 & 1 & 1 \\
\hline \multicolumn{5}{|l|}{ Physical workload } \\
\hline Over loaded & 35 & 77 & $3.64[1.22,9.29]^{*}$ & $2.13[0.43,10.50]$ \\
\hline Equally loaded & 21 & 225 & $0.69[0.25,1.95]$ & $0.74[0.14,3.71]$ \\
\hline Less than others & 5 & 37 & 1 & 1 \\
\hline \multicolumn{5}{|l|}{ Malaria infection history } \\
\hline Yes & 30 & 49 & $5.73[3.19,10.29]^{*}$ & $3.50[1.40,8.58]^{*}$ \\
\hline No & 31 & 290 & 1 & 1 \\
\hline
\end{tabular}

*statistically significant variables at $\mathrm{p}<0.05,95 \% \mathrm{CI}$-confidence interval

Note: All variables with the $\mathrm{p}<0.25$ in the bivirate was included in the multivariate to be analyzed 


\section{Discussion}

In the present study, the overall prevalence of anemia among adolescents' girls was $15.2 \%$. This was 2 in 10 adolescent, showed that there is substantial burden of Anaemia in the district. The figure might have been underestimated as the study was conducted in January - a relatively food surplus season. Furthermore, the effect of seasonal fluctuations of other risk factors e.g. malaria might not been captured due to the cross-sectional nature of the study. The present finding is comparable with the previous estimated prevalence of anaemia for Ethiopia among adolescent and young female $15 \%$ [10] and National prevalence in Ethiopia, 15\% [13].

The present finding is higher than study report from Tigray, Ethiopia 7.1\% [12], prevalence of Anaemia was 14\% [22]. On other side, the current finding is lower than study report of Maharashtra, India 87\% [23], rural adolescent 75\% [24]; in Bangladesh 51.6\% [25]; in Tertiary Care Hospital 50\% [26]; Jimma, South West Ethiopia 37.6\% [27]; South west of Ethiopia 28\% [6]; and Afar, North East Ethiopia $22.8 \%$ [29]. The reason for variation might be socioeconomical and geographical differences contributed.

In this study, the majority of anaemia cases $13.7 \%$ were mild (Hgb level $<12 \mathrm{~g} / \mathrm{dl}$ ) followed by $1.5 \%$ of moderate anaemia $(7-9.9 \mathrm{~g} / \mathrm{dl})$ and a similar condition was observed in Jimma, Ethiopia, in which majority of the cases had moderate anaemia (19.6\%), mild anemia (18.1\%) and no severe case [27]. Study in India Aurangabad district, Maharashtra showed similar, there was no severe case detected [30].

On other hand, by taking the WHO reference standard, -2 SD ( $\mathrm{Z}$ score) as a cutoff point [18]. In this present study, adolescent girls showed evidence of stunting on the basis of height-for-age $<-2$ SD ZScore. The present prevalence of stunted and thinness were account as $21.3 \%$ and $16.5 \%$, respectively. This finding is similar with the study report of nutritional status of adolescent girls in thinness $21.6 \%$ and stunting $15 \%$ [31]. On other side, the study report from Chiro, West Hararge. Ethiopia stunting and underweight was $7.2 \%$ and $24.4 \%$ [32]; in Urban Slums of Dibrugarh was $31.33 \%$ and $25.7 \%$ [33] respectively and study finding in Jimma Zone, South West Ethiopia were showed that $83.8 \%$ and $16.0 \%$ of underweight and stunting, respectively [34]; in Raipur city Chhattisgarh, India was $53.8 \%$ [35] and also adolescent stunting was reported $18.6 \%$ [36].

Nutritionally adolescence is critical life period; hence, adolescent girls are advised to eat diversified diet than usual. However, this was not the case in the study area, the level of diet diversity among this study participant was lower and it was significantly associated with anaemia in this study. This study report is agreed with the study finding West Uganda [37].

Malaria attack history in the last 3 month duration was the factor that revealed a significantly association with occurrence of anaemia in adolescent girls $[\mathrm{AOR}=3.5]$, this finding is similar to the study conducted in Gonder, North West Ethiopia, malaria attack history was 13.28 times odds of increased to develop anaemia [38] and Western Uganda, anemia was positively correlated with malaria [37]. The reason might be failure to seek early diagnosis and treatment of malaria that might lead to increased RBC destruction and consequently, end up in anaemia.

In our study, long duration of menstruation (more than five days) among adolescent girls significantly associated with anaemia $[\mathrm{AOR}=5.10]$. This is consistent with the study conducted elsewhere in Bangladesh, Tangail region, menstrual duration 5 days or more was risk factor for anemia [39] India, menstrual cycle lasting 7 or more days was important contributing factors for anemia [40]. Cooke et al [41] and Power et al [42] also witness adolescent girls with heavy menstrual bleeding can cause anaemia. This is likely due to the blood loss secondary to long menstrual duration.

In the present study, there was no significant association found between the age of adolescent, educational status of adolescent, educational status of father and mother, marital status of adolescent girls, occupation of father and mother, family size and anaemia in this study. This result was similar with the study finding [28].

\section{Conclusion}

The current finding reported substantial burden of anaemia (15.2\%) and under nutrition (21.6 thinness and 15\% stunting) among adolescent girls in the study area. Malaria attack history, low dietary diversity, long menstrual duration and less frequent consumption of legumes identified as contributing factors for adolescent anaemia. To make significant effect, it needs strong integrative strategies, policy makers, governmental and NGO intervention to tackle this public health burden.

\section{Availability of Data and Materials}

We have data already collected from community and entered SPSS and analyzed. So we declare an Availability of data and materials.

\section{Competing Interest}

The authors declare that they have no competing interest.

\section{Funding}

No fund source for this particular study

\section{Authors' Contributions}

The authors participated in the preparation of the study design, data collection, analysis and interpretation of data. Authors read and approved the final manuscript.

\section{Acknowledgements}

The authors would like to appreciate mothers participated in this study and also extend warm thanks for data collectors 
who took part, finally we would like thanks to Hadero Tunto zuria woreda health office permitted for data collection at community.

\section{References}

[1] WHO. Strategic directions for improving Adolescent Health in South-East Asia region. Geneva: WHO; 2011.

[2] United Nation Children's Fund. The state of the world children: adolescent an age of opportunity. New York: UNICEF; 2011.

[3] WHO. Prevention of Iron deficiency Anaemia in adolescents: Role of weekly iron and folic acid supplementation. India: WHO; 2011.

[4] WHO. Global Accelerated Action for the Health of Adolescent (AA-HA!): Guidance to support country implementation. Geneva: World Health Organization; 2017.

[5] Chandrakumari AS, Sinha P, Singaravelu S, and Jaikumar S. Prevalence of anaemia among adolescent girls in a rural area of Tamil Nadu, India. Journal of family med prim care $2019 ; 8$ (4): 1414-1417.

[6] Wolde T, Amanu W, Mekonnin D, Yitayin F, Abu F, Dufera F, Birhanu T, and Ejeta E. Nutritional status of adolescent girls living in South west of Ethiopia: Food Science and Quality Management 2014; 4: 58-64.

[7] WHO. WHA Global Nutrition Targets 2025: Anaemia Policy Brief. Geneva: WHO; 2014.

[8] Lopez A, Cacoub P, Macdougall IC and Peyrin-Biroulet L. Iron deficiency anaemia. Lancet 2016; 387: 907- 916.

[9] Christopher V, Charles, Maddock J (Ed.). Iron deficiency anemia: A Public Health Problem of Global Proportions. Public Health Methodology, Environmental and Systems Issues 2012; 121-122.

[10] Yasutake S, He H, Michele R, Decker, Freya L. Sonenstein Nan M, Astone. Anemia among adolescent and young women in Low-and-Middle-income countries. International Journal child Health and Nutrition 2013; 2: 105-112.

[11] Hadero, Hadero Tunto Zuria District Health Office. Woredabased health sector annual Plan. Hadero: HTZ; 2016.

[12] Mulugeta A, Gebre M, Abdelkadir M, Tsadik AG, Yesus AG and Stoecker BJ. Iron deficiency in adolescent school girls from Tigray, Northern Ethiopia. Journal Fed Amer Soci Exper Bio 4; 2010.

[13] Central Statistical Agency (CSA) Ethiopia and ICF. Ethiopia demographic and Health survey 2012. Addis Ababa, Ethiopia, and Rockville, Maryland, USA: CSA and ICF; 2012.

[14] Swindale A, Bilinsky P. Household dietary diversity score (HDDS) for Measurement of household food access: indicator guide: $\quad$ FANTA III; 2006 www.fantaproject.org/downloads/pdf/HDDs_v2pdf.

[15] Food and Agriculture Organization of the United Nations. Guidelines for measuring household and individual dietary diversity. Rome: FAO; 2011.

[16] Hemocue $^{\mathrm{R}} 301$ user manual; 2015 (accessed 2016).
[17] WHO. Multicenter Growth Reference Study Group: WHO child growth standards: length/height-for-age, weight-for-age, weight-for-length, weight-for-height and body mass index-forage: Methods and development. Geneva: World Health Organization; 2006 [Accessedin Nov 8, 2016].

[18] De-onis, M, Onyango, AW, Borghi, E, Siyam, A, Nishida, C, Siekmann, J. Development of a WHO growth reference for school-aged children and adolescents. Bulletin of world health organization 2007, 85 (9).

[19] World Health Organization. WHO AnthroPlus for personal computers manual: Software for assessing growth of the world's children and adolescents. Geneva: WHO; 2009 [Accessed in Feb 12, 2016]. Available in: http://www.who.int/growthref/tools/en/

[20] International Nutritional Anemia Consultative Group (INACG). Adjusting Hemoglobin value in program surveys, 2002.[Accessed on April 05, 2016].

[21] WHO. The global prevalence of anaemia in 2011. Geneva: World Health Organization; 2015.

[22] Andriastuti M, Ilmana G, Nawangwulan SA, and Kosasih KA. Prevalence of anemia and iron profile among children and adolescent with low socio-economic status. International Journal of Pediatrics and Adolescent Medicine/Elsevier 2019; $1-5$.

[23] Ahankari AS, Myles PR, Fogarty AW, Dixit JV, and Tata LJ. Prevalence of iron deficiency Anaemia and risk factors in 1010 adolescent girls from rural Maharashtra, India: a crosssectional survey. Public Health Elsevier 2016; 1-8.

[24] Baliga, SS, Naik, VA, and Mallapur, MD. Nutritional status of adolescent girls residing in rural area: A community-based cross-sectional study. Journal of Soc 2014; 41 (1): 22-25.

[25] Mistry SK, Jhohura T, Khanam F, Akter F, Khan S, Yunus FM, Hossain MB, Afsana K, Haque MR, and Rahman, M. An outline of anemia among adolescent girls in Bangladesh: findings from a cross-sectional study. BMC Haematology 2017; 17 (13): 1-9.

[26] Kumari, R, Bharti, RK, Singh, K, Sinha, A, Kumar, S, Saran, A, and Kumar, U. Prevalence of Iron Deficiency and Iron Deficiency Anaemia in Adolescent Girls in a Tertiary Care Hospital. Journal of clinical and Diagnosis Research 2017; 11 (8): 4-6.

[27] Assefa S, Mossie A and Hamza L. Prevalence and severity of anemia among school children in Jimma town, southwest Ethiopia. BMC Hematology Journal 2014; 14: 3.

[28] Gebremedhin S, Enquselassie F and Umeta M. Prevalence and Correlates of maternal anemia in ruralSidama, Southern Ethiopia. Africa Journal repro health 2014; 18 (1): 53.

[29] Adem OS, Tadsse K, and Gebremedhin A. Iron deficiency anemia is moderate Public health problem among school going adolescent girls in Berahle district, Afar, North East Ethiopia. Journal Food and Nutrition Science 2015; 3 (1); 1016. Doi: 10.11648/jfns.20150301.12.

[30] Jagannath DK, Kuldeep DS, Narendra SM, Hrishikesh AK, and Vivek SB. 2014. Prevalence and Socio-demographic factors related to anemia among adolescent girls in a rural area of Aurangabad district, Maharashtra. Intern journal science Study 2014; 2 (8). 
[31] Teji K, Dessie Y, Asseba T and Abdo M. Anaemia and Nutritional status of adolescent girls in Babile district Eastern, Ethiopia. Pan Africa Medical Journal 2016; 1-10.

[32] Damie TD, Wondafras M, and Nigussie A. Nutritional status and associated factors among school adolescent in Chiro Town, West Hararge, Ethiopia. European Journal of Therapeuticus 2016; 21: 32-42.

[33] Bhattacharyya $\mathrm{H}$ and Barua A. Nutritional status and Factors affecting nutrition among adolescent girls in Urban Slums of Dibrugarh, Assam. Nat journal community med 2013; 4 (1): $35-9$.

[34] Assefa H, Belache T, and Negash L. Socioeconomic factors associated with underweight and stunting among adolescents of Jimma Zone, South West Ethiopia: A Cross-Sectional Study. Journal Public Health 2013; 7. http://dx.doi.org/10.1155/2013/238546

[35] Patanwar P and Sharma K. Nutritional status of Kurmi Adolescent girls of Raipur city Chhattisgarh, India. International journal scientific and Research publication 2013; 3 (11): 1-6.

[36] Berget T, Magala-Nyago C and Iversen PO. Nutritional Status among adolescent girls in children's homes: Anthropometry and dietary patterns. Clinical Nutrition Elsevier 2017; 1-8.

[37] Barugahara EI, Kikafunda J, and Gakenia, WM. Prevalence and risk factors of nutritional anemia among female school children in Masindi district, Western Uganda. Africa Journal Food Agri, Nutr and Develop 2013; 13 (3).

[38] Alem M, Enawgaw B, Gelaw A, Kena T, Seid M, and Olkeba Y. Prevalence of anemia and associated risk factors among pregnant women attending antenatal care in Azezo Health Center Gondar town, Northwest Ethiopia. Journal Inter discipl Histopathol 2013; 1 (3): 137-144.

[39] Miah S, Rahman N, Prodhan UK, Linkon MR, Madumita, and Rahman, S. Prevalence of iron deficiency of anemia among adolescent girls and it risk factor in Tangail region of Bangladesh. IJRET 2014; 3 (06).

[40] Arun V, Panat, Sambhji A, Pathare, Asran S, and Rohokale G. Iron deficincy among rural college girls: result of poor nutrition and prolonged menstruation. Journal community Nutrition health 2013; 2 (2).

[41] Cooke AG, McCavit TL, Buchanan, GR, and Powers, JM. Iron Deficiency Anemia in Adolescents Presenting with Heavy Menstrual Bleeding. Journal of Pediatric and Adolescent Gynecology 2016; 1-18.

[42] Power JM, Stanek JR, Srivaths L, Haamid FW, and Brien SH. Hematologic Considerations and Management of Adolescent Girls with Heavy Menstrual Bleeding and Anemia in US Children's Hospitals. Journal of paediatric and Adolescent Gynecology 2018; 31 (5): 446-450. 